\section{Cytomegalovirus antiviral drug resistance: future prospects for prevention, detection and management}

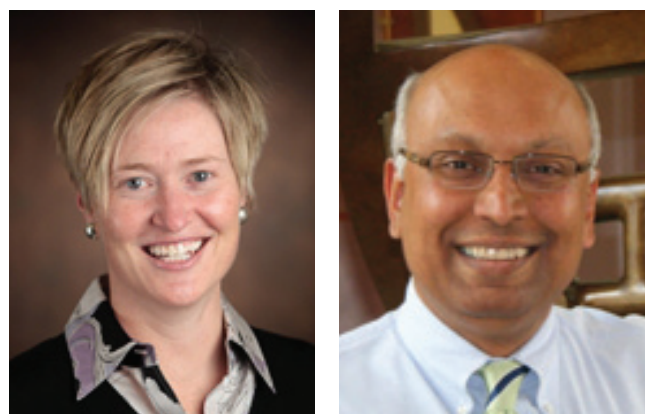

"Antiviral drug resistance is a dreaded complication because it makes treatment more difficult, is associated with poor clinical outcomes and may lead to allograft loss or death.”

Kimberly E Hanson ${ }^{1,2}$ \& Sankar Swaminathan ${ }^{*, 1}$

Human cytomegalovirus (CMV) is one of the most important viral pathogens affecting hematopoietic stem cell transplant (HSCT) and solid organ transplant (SOT) recipients, because infection is frequent and it exerts short- and long-term effects on host immunity. The direct effects of active CMV infection include fever, bone marrow suppression and invasive end organ disease. Indirectly, CMV increases risk for other infections and is associated with increased rejection, graft loss, mortality and transplant costs [1]. Given the impact of CMV on transplant outcomes, prevention of active infection and aggressive treatment of invasive disease are essential.

Prevention strategies include universal antiviral prophylaxis or pre-emptive therapy that is based on sequential viral load monitoring in blood. Both approaches prevent CMV disease in at-risk patients, but they are limited by the toxicities of available anti-CMV drugs (i.e., cytopenias from valganiclovir, ganciclovir and cidofovir, and nephrotoxicity from foscarnet and cidofovir) and the potential for the virus to develop antiviral drug resistance (AVR).
Furthermore, optimal viral load thresholds for the initiation of pre-emptive therapy have not been defined and it is likely that these cut-offs will vary based on host factors as well as the quantitative PCR assay used for testing. Due to these complexities, an ideal preventative approach has yet to be identified. Only prophylaxis would be expected to prevent CMV indirect effects, but early treatment of viremia (i.e., pre-emptive therapy) may allow some reconstitution of protective immunity.

\section{Current approaches}

HSCT programs generally apply preemptive therapy to avoid the marrow suppressive effects of valganciclovir (VGCV) or ganciclovir (GCV). Some centers also utilize prophylaxis for unrelated, HLAmismatched or cord blood transplants. SOT programs also typically use prophylaxis for the highest risk patients (CMV seropositive donor $[\mathrm{D}+] / \mathrm{CMV}$ seronegative recipient [R-]), and may consider pre-emptive approaches for moderate-risk $\mathrm{R}+$ liver or kidney recipients. The routine use of prolonged CMV prophylaxis (i.e., $\geq 6$ months of VGCV) has raised concern about AVR,

\section{KEYWORDS}

- adoptive immunotherapy

- antiviral drug resistance

- cytomegalovirus • human CMV 
“Three experimental

human cytomegalovirus antivirals have entered

Phase II and III clinical trials.” with the potential to develop cross-resistance to currently available drugs. A recent report also described a high incidence of AVR among D+/Rkidney recipients receiving pre-emptive therapy [2]. AVR is a dreaded complication because it makes treatment more difficult, is associated with poor clinical outcomes and may lead to allograft loss or death [3].

GCV and its oral prodrug VGCV are the current cornerstones of CMV therapy. These agents are nucleoside analogs that require phosphorylation by the viral protein kinase (UL97 gene) in order to inhibit the CMV DNA polymerase (UL54 gene) that is required for viral replication. Certain mutations within the UL97 and/ or UL54 genes have been linked to GCV and VGCV resistance (reviewed in [4]). Cidofovir (CDV) and foscarnet (FOS) act at the level of the DNA polymerase. Mutations in the UL54 gene can confer resistance to CDV, FOS or crossresistance to both CDV and GCV. CDV resistance has been reported following GCV therapy, while FOS resistance typically requires previous exposure to that drug.

CMV AVR is suspected when viral load increases or plateaus despite at least 2 weeks of appropriate antiviral therapy. Drug resistance is then confirmed by detecting specific gene mutations or with phenotypic drug susceptibility testing. Most laboratories use standard Sanger DNA sequencing of known resistance loci to detect drug resistance mutations. Confirmation of genotypic resistance is preferred because host factors or inadequate drug levels may also affect treatment response, and empiric changes in antiviral therapy often have adverse side effects. A limitation of current laboratory practice is that standard DNA sequencing may not detect drugresistant heterogeneity $(<20-30 \%)$ in a mixed viral population. Minority CMV populations can be clinically relevant and newer technologies such as next-generation sequencing [5], that can detect subpopulations on the order of $1-3 \%$, are being explored for the enhanced detection of genotypic resistance.

Most AVR has been reported in the context of long-term GCV or VGCV use, usually after at least 6 weeks of drug exposure [6]. Precise estimates of AVR prevalence and risk in an era of VGCV prophylaxis and pre-emptive therapy are limited by the paucity of large prospective studies. In general, CMV AVR appears to be relatively uncommon after HSCT [7] but may affect as many $5-27 \%$ of viremic SOT recipients, with the highest incidence following lung transplantation [8]. Risk factors for AVR include D+/Rserostatus in SOT, lung transplantation, high viral load, persistent viremia on therapy and suboptimal GCV concentrations [9], as well as haploidentical T-cell-depleted HSCT [10]. The exact role, if any, that therapeutic drug monitoring should have in detecting subtherapeutic GCV drug concentrations needs to be better defined.

\section{Future possibilities}

Given the safety profile of traditional drugs in conjunction with the problem of AVR, bettertolerated anti-CMV agents with different mechanisms of action are needed. The primary risk for active infection after transplantation is a reduced number and function of CMV-reactive $T$ cells. Therefore, reduction in immunosuppression when feasible is an essential component of CMV management.

Adoptive transfer of donor-derived or third party $\mathrm{T}$ cells is being explored as a way to promote immune reconstitution and reduce viral burden. Perhaps the most exciting approach is the generation and expansion of broad-spectrum, cytotoxic donor $\mathrm{T}$ lymphocytes in vitro which recognize virus-specific epitopes, persist and proliferate in vivo [11]. These strategies have been used in prophylactic and pre-emptive regimens as well as for the treatment of clinically resistant infection. Despite significant heterogeneity, most studies have observed that CMV-specific T-cell immunity can be restored without inducing graft-versus-host disease or other significant toxicity [12-14]. It is possible that adoptive T-cell therapy could be used in combination with shorter course antiviral prophylaxis to enhance cellular immune protection while minimizing antiviral drug side effects, cost and AVR.

Three experimental CMV antivirals have entered Phase II and III clinical trials. Maribavir (MBV) and letermovir (LTV) have unique mechanisms of action. MBV is an oral UL97 protein kinase inhibitor that initially failed Phase III prophylaxis trials possibly due to inappropriate dose selection [15]. More recently, two as of yet unpublished Phase II dose-ranging studies have been completed that examined higher doses of MBV as pre-emptive therapy (EuraCT: 2010-024247-32) and for refractory or resistant CMV (ClinicalTrials.gov identifier: NCT01611974). Since UL97 is needed to phosphorylate GCV, therapy with MBV and GCV would be expected to be antagonistic. 
However, the UL97 mutations elicited by MBV and GCV are different and MBV retains in vitro activity against most GCV- and CDV-resistant viruses [16]. Of note, there is a single p-loop mutation (F342S) that has been shown to confer dual cross-resistance between MBV and GCV, but does not affect kinase activity or growth in cell culture [17]. The F342S mutation has not yet been observed in vivo.

LTV (formerly AIC-246) is a CMV-specific viral terminase inhibitor with potent in vitro activity against wild-type and drug-resistant virus. Oral and intravenous formulations have been developed, which is of potential benefit for patients unable to tolerate or absorb oral therapy. LTV recently entered Phase III prophylaxis studies (ClinicalTrials.gov identifier: NCT02137772) after successful completion of an HSCT Phase II dose-escalation trial [18]. No AVR information was provided in the Phase II report. However, high-grade resistance mutations in the UL56 terminase gene are readily selected in vitro and await clinical correlation [19]. The potentially low barrier to LTV resistance may limit the utility of this agent as monotherapy for severe disease or high-grade viremia.

Brincidofovir (formerly CMX-001) is a broadspectrum, orally bioavailable lipid conjugate of CDV. Unlike CDV, brincidofovir (BCV) is not a substrate for human organic anion transporters (hOATs) and therefore has reduced potential to cause renal impairment. A Phase II prophylaxis study in HSCT was completed wherein no development of AVR was noted [20]. Phase III trials are currently underway (ClinicalTrials.gov identifier: NCT0176170). Despite overlapping resistance mechanisms, BCV may have a role in treating genotypically resistant infection because the drug has potent in vitro activity and achieves high intracellular concentrations.

Combination antiviral therapy is the standard of care for other viral infections, such as HIV and HCV. Dual DNA polymerase inhibitor treatment for $\mathrm{CMV}$, however, has been limited by additive toxicity, cross-resistance and a lack of evidence to support effectiveness. We have now entered an exciting time of late-phase drug development for several anti-CMV agents with novel drug targets, potent activity and reduced toxicity. Additionally, CMV-specific immunotherapies are under active investigation. These agents used alone or in combination, create new opportunities to refine our management strategies and potentially provide therapeutic alternatives for drug-resistant CMV. Studies are now needed to determine whether combinations of the new agents with different mechanisms of action can improve treatment efficacy and/or reduce the risk for AVR. Additionally, studies comparing prophylactic versus pre-emptive approaches, that utilize standardized CMV assays and are powered to assess both the direct as well as indirect effects of CMV, will better inform clinical practice in the future.

\section{Financial \& competing interests disclosure}

The authors have no relevant affliations or financial involvement with any organization or entity with a financial interest in or financial conflict with the subject matter or materials discussed in the manuscript. This includes employment, consultancies, honoraria, stock ownership or options, expert testimony, grants or patents received or pending, or royalties.

No writing assistance was utilized in the production of this manuscript.
"We have now entered an exciting time of latephase drug

development for several anti-cytomegalovirus agents with novel drug targets, potent activity and reduced toxicity."

\section{References}

1 Roman A, Manito N, Campistol JM et al. The impact of the prevention strategies on the indirect effects of CMV infection in solid organ transplant recipients. Transplant. Rev. 28(2), 84-91 (2014).

2 Couzi L, Helou S, Bachelet T et al. High incidence of anticytomegalovirus drug resistance among $\mathrm{D}+\mathrm{R}$ - kidney transplant recipients receiving preemptive therapy. $\mathrm{Am}$. J. Transplant. 12(1), 202-209 (2012).

3 Eid AJ, Arthurs SK, Deziel PJ, Wilhelm MP, Razonable RR. Emergence of drug-resistant cytomegalovirus in the era of valganciclovir prophylaxis: therapeutic implications and outcomes. Clin. Transplant. 22(2), 162-170 (2008).

4 Lurain NS, Chou S. Antiviral drug resistance of human cytomegalovirus. Clin. Microbiol. Rev. 23(4), 689-712 (2010).

5 Sahoo MK, Lefterova MI, Yamamoto F et al. Detection of cytomegalovirus drug resistance mutations by next-generation sequencing. J. Clin. Microbiol. 51(11), 3700-3710 (2013).

6 Kotton CN, Kumar D, Caliendo AM et al. Updated international consensus guidelines on the management of cytomegalovirus in solid-organ transplantation. Transplantation 96(4), 333-360 (2013).
7 van der Beek MT, Marijt EW, Vossen AC et al. Failure of pre-emptive treatment of cytomegalovirus infections and antiviral resistance in stem cell transplant recipients. Antiviral Ther. 17(1), 45-51 (2012).

8 Limaye AP. Ganciclovir-resistant cytomegalovirus in organ transplant recipients. Clin. Infect. Dis. 35(7), 866-872 (2002).

9 Le Page AK, Jager MM, Iwasenko JM, Scott GM, Alain S, Rawlinson WD. Clinical aspects of cytomegalovirus antiviral resistance in solid organ transplant recipients. Clin. Infect. Dis. 56(7), 1018-1029 (2013). 


\section{EDITORIAL Hanson \& Swaminathan}

10 Shmueli E, Or R, Shapira MY et al. High rate of cytomegalovirus drug resistance among patients receiving preemptive antiviral treatment after haploidentical stem cell transplantation. J. Infect. Dis. 209(4), 557-561 (2014).

11 Papadopoulou A, Gerdemann U, Katari UL et al. Activity of broad-spectrum $T$ cells as treatment for AdV, EBV, CMV, BKV, and HHV6 infections after HSCT. Sci. Translat. Med. 6(242), 242ra283 (2014).

12 Schmitt A, Tonn T, Busch DH et al. Adoptive transfer and selective reconstitution of streptamer-selected cytomegalovirus-specific $\mathrm{CD}^{+} \mathrm{T}$ cells leads to virus clearance in patients after allogeneic peripheral blood stem cell transplantation. Transfusion 51(3), 591-599 (2011).

13 Uhlin M, Gertow J, Uzunel M et al. Rapid salvage treatment with virus-specific $\mathrm{T}$ cells for therapy-resistant disease. Clin. Infect. Dis. 55(8), 1064-1073 (2012).

14 Leen AM, Bollard CM, Mendizabal AM et al. Multicenter study of banked third-party virus-specific $T$ cells to treat severe viral infections after hematopoietic stem cell transplantation. Blood 121(26), 5113-5123 (2013).

15 Marty FM, Boeckh M. Maribavir and human cytomegalovirus-what happened in the clinical trials and why might the drug have failed? Curr. Opin. Virol. 1(6), 555-562 (2011).

16 Drew WL, Miner RC, Marousek GI, Chou S. Maribavir sensitivity of cytomegalovirus isolates resistant to ganciclovir, cidofovir or foscarnet. J. Clin. Virol. 37(2), 124-127 (2006).
17 Chou S, Ercolani RJ, Marousek G, Bowlin TL. Cytomegalovirus UL97 kinase catalytic domain mutations that confer multidrug resistance. Antimicrob. Agents Chemother. 57(7), 3375-3379 (2013).

18 Chemaly RF, Ullmann AJ, Stoelben $\mathrm{S}$ et al. Letermovir for cytomegalovirus prophylaxis in hematopoietic-cell transplantation. N. Engl. J. Med. 370 (19), 1781-1789 (2014).

19 Goldner T, Hempel C, Ruebsamen-Schaeff H, Zimmermann H, Lischka P. Geno- and phenotypic characterization of human cytomegalovirus mutants selected in vitro after letermovir (AIC246) exposure. Antimicrob. Agents Chemother. 58(1), 610-613 (2014).

20 Marty FM, Winston DJ, Rowley SD et al. CMX001 to prevent cytomegalovirus disease in hematopoietic-cell transplantation. N. Engl. J. Med. 369 (13), 1227-1236 (2013). 